\section{LAS MALLAS ESPACIALES Y SU APLICACION EN CUBIERTAS PARA ESTADIOS}

\author{
Juan Martínez Apezteguia \\ Dr. Ingeniero Industrial \\ Director Técnico de ORONA, S. COOP. \\ División Estructuras
}

\section{Introducción}

Durante las últimas décadas el empleo de las estructuras espaciales en la construcción ha tenido un espectacular desarrollo. Entre las causas que lo han motivado cabria citar como fundamental el hecho de que están concebidas bajo la perspectiva de conseguir un alto grado de fabricación, aspecto que ha permitido importantes ahorros de mano de obra tanto en fabricación como en montaje. La complejidad del cálculo requerido para su dimensionamiento constituyó durante muchos años una importante limitación en sus aplicacio nes. La generalización del empleo de los ordenadores ha permitido el análisis rápido y preciso de estas estructuras propiciando grandemente su competitividad.

Por sus singulares caracteristicas las mallas espaciales son especialmente aptas para cubrir grandes espacios in columnas intermedias. De aquí que se hayan aplicado con gran profusión como estructuras portantes en las cubiertas de recintos deportivos cerrados.

Las cubiertas para tribunas de estadios deportim vos, si bien presentan caracteristicas muy diferentes de las empleadas sobre recintos cerrados, por sus especiales condiciones de apoyo suelen imponer unos diseños estructurales sometidos a imporm tantes solicitaciones. Esto invita a pensar en la utilización de las estructuras espaciales con este fin.

Con este documento se pretende en primer lugar exponer los aspectos técnicos más característicos de este tipo de estructuras, para juzgar posteriormente sobre la conveniencia de aplicación de las mismas a las cubiertas de estadios.

\section{Descrippción y características}

Desde un punto de vista de clasilicación estructum ral puede considerarse espacial toda estructura en la que la distribución de los elementos resistentes es tal que no permite un análisis de su comportamiento por planos independientes. En contraposición, las estructuras consideradas como planas serían aquellas susceptibles de ser descompuestas en diferentes subconjuntos comprendidos, cada uno de ellos, en un plano dentro del cual pueden ser analizados separadamente de los demás. En esta clasificación quedan incluidas las formas estructurales un tanto singulares como cúpulas, bóvedas hiperboloides, etc., asi como otras muchas soluciones constructivas, algunas incluso muy elementales.

Dentro de las estructuras espaciales, las que han sido objeto del gran desarrollo a que se ha hecho referencia en la introducción, son un determinado tipo de estructuras, que propiamente deberian ser consideradas como celosias tridimensionales y que con frecuencla se conocen por mallas estéreas o espaciales. Estas estructuras se caracterizan por estar constituidas a base de elementos muy simples (barras y nudos) que ordinariamente se ensamblan en obra por atornillado para formar el conjunto estructural. La topología, distribución geométrica de barras en el espacio, se consigue como consecuencia de la repetición y yuxtaposición de poliedros sencillos correspondiéndose las barras con las aristas de aquéllos y los nudos con sus vértices. El conjunto, de gran repetibilidad geométrica, recuerda en cierto modo, y no por

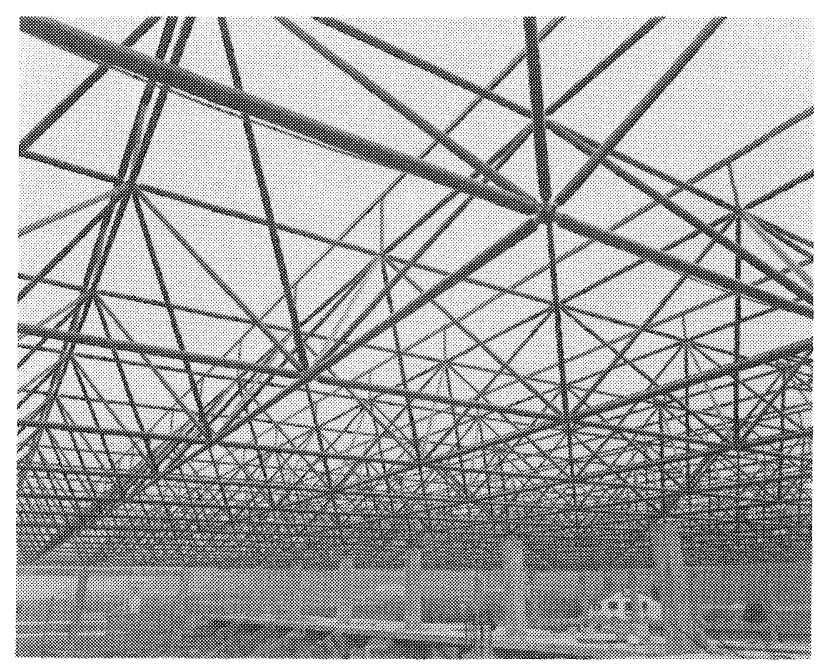


casualidad, las organizaciones estructurales más elementales de la naturaleza como son las que forman los átomos para componer los cristales moleculares. En los casos más rrecuentes el conjunto está comprendido entre dos superficies en volventes, sensiblemente parelelas, planas o no, que se denominan capas de la malla. Cada capa suele estar compuesta por una red de barras dism puestas en forma de reticula. Los nudos de cada capa se conectan con los de la opuesta mediante otras barras denominadas normalmente "diago nalesn.

Con el fin de presentar las peculiaridades de estas estructuras, a continuación se exponen los aspectos comparativos que, a nuestro juicio, mejor diferencian las mallas espaciales respecto de las estructuras metálicas convencionales.

Las primeras diferencias se deducen de la propia organización geométrica. Las estructuras conven cionales para cubrir grandes luces ordinariamente se componen de subestructuras planas de diferentes ordenes (Iácenas, cerchas, correas, etc.), cada una de las cuales recibe las cargas de otras de orden inmediato inferior para transmitilas, a su vez, a las de orden superior. En las mallas espa clales, por el contrario, no existen elementos preponderantes. Todas las barras forman parte de un conjunto entrelazándose de forma que los esfuerzos se reparten entre todas siguiendo preferentemente las direcciones principales de la reticula. En las aplicaciones más normales de estas estructuras, las superficies envolventes de la malla son dos planos paralelos que recuentemente se dism ponen horizontalmente dando lugar a lo que se conoce por "cubiertas planas". Conviene aclarar que a pesar de esta denominación se trata de es. tructuras espaciales, que en absoluto se compo nen de subestructuras planas. El comportamiento conjunto es similar al de una placa en la cual las flexiones según dos direcciones ortogonales que dan canalizadas a través de las barras horizontales y los esfuerzos cortantes son soportados por las diagonales. Normalmente las conexiones entre las barras son tales que permiten ser consideradas como articulaciones puras; en consecuencia dim chas barras, a menos que reciban acciones directas del exterior, quedan exclusivamente sometidas a esfuerzos axiles; de aqui su clasificación como celosias.

El trabajo conjunto de todos los elementos y el reparto de los esfuerzos, en varias direcciones principales, hace que los valores máximos de las solicitaciones sean normalmente muy inferiores a los que se producirian en una estructura equivalente constituida por celosías planas. De aquí se deduce una de las aptitudes más significativas de las mallas, que es su capacidad para cubrir mayor res luces bajo los mismos niveles de esfuerzo. Teniendo en cuenta además que sus barras solo trabalan a traccion o compresión y que ordinariamente se construyen con perfil tubular, optimo para este tipo de solicitaciones, se comprende fám climente que estas estructuras tengan un peso proplo muy inferior al de las convencionales. Es frecuente que para cublertas ligeras con luces comprendidas entre 20 y $50 \mathrm{~m}$ el peso propio osclle entre 12 y $20 \mathrm{~kg} / \mathrm{m}^{2}$.

Otra consecuencia de la ravorable distribución de estuerzos es la obtención de estructuras muy esbeltas, en el sentido de que el volumen ocupado es minimo. Una caracteristica interesante a considerar en cualquier estructura de cubierta es la relación entre el canto y la luz. Normalmente es conveniente obtener valores reducidos de este co ciente. La disminución del mismo hace crecer inversamente las solicitaciones y las deflexiones bajo carga, exigiendo finalmente dimensionamientos más costosos. A nivel práctico el citado cociente suele quedar limitado, y en el caso de las estructuras metalicas ordinarias para grandes luw ces no conviene adoptar valores inferiores a 1/15 aproximadamente. En las mallas espaciales el canto suele denominarse "espesor de malla", siendo la separación entre las capas opuestas. En aplicaciones a cubiertas planas apoyadas en todo el contorno puede llegarse razonablemente a relam ciones espesor/luz del orden de $1 / 20 \mathrm{sin}$ esfuerzos ni deflexiones excesivos.

Evidentemente el óptimo aprovechamiento de estas estructuras se consigue cuando las diferentes direcciones principales de la malla contribuyen equilibradamente a soportar las cargas exteriores. Esto se consigue en mayor grado en aquellas cubiertas en que la malla puede apoyarse en todo el contorno y cuando las dimensiones generales que definen la planta son parecidas. Es decir, en este sentido será más favorable una cubierta de planta cuadrada, que una rectangular de la misma luz pero con notable diferencia entre sus dimensiones principales. Análogamente una planta circular se comportaria mejor que otra eliptica de gran excentricidad. En definitiva, la competitividad de las mallas espaciales respecto de las estructuras pla nas depende no sólo de la luz o dimensión mini. ma entre alineaciones de soportes, sino tambièn de las proporciones de la planta a cubrir. Por sum puesto que si en una cubierta cuadrada la malla sólo puede soportarse en dos contornos opuestos, quedando los otros dos sin apoyos, una de las direcciones no contribuye al esfuerzo y el beneficio derivado del aprovechamiento de la forma se desaprovecha. En tal caso la malla queda trabaw jando como un conjunto simple de vigas paralelas. 


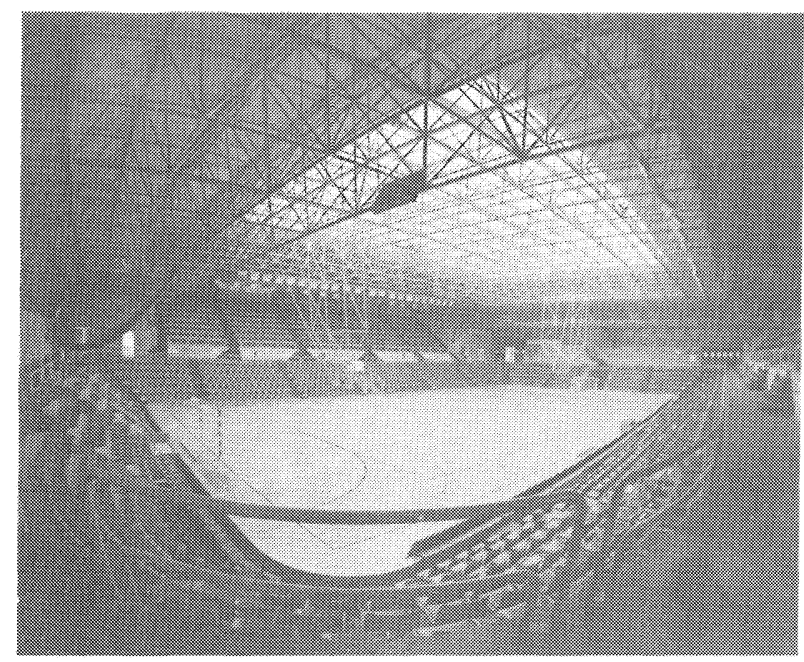

Otra caracteristica destacable es la rigidez del conjunto estructural que forman las mallas espam ciales. La cubierta se comporta como un entrama do sólido prácicamente indeformable que le conflere importantes ventajas. En primer lugar permite una distribución muy lexible de los apoyos o ca nexiones con la sustentación, requiriendo generalm mente un menor número de columnas y permitiendo además una mayor versatllidad en cuanto a las posiciones de las mismas. Como ejemplo cabe cim tar la cubierta del Polideportivo Municipal de Bur gos cuya planta supera los $4.000 \mathrm{~m}^{2}$ : sustentándo se exclusivamente en cuatro puntos.

Pero, además, el hecho de que la propia cubierta se comporte como una placa uniendo las cabezas de las columnas sobre las que descansa, hace que todas ellas rabajen conjuntamente cuando la estructura queda somelida acciones exteriores horizontales. Esio reporta un nuevo beneficio sa bre el dimensionamiento de la sustentación respecto de las acciones de viento y esfuerzos sismi $\cos$.

Un aspecto dependiente de la interrelación entre todos los elementos de la estructura es la necesidad de calcularla toda ella conjuntamente. En el caso de que la estructura tenga un comportamiento lineal, el modelo matemático que la representa implica el establecimiento de un sistema de ecuam ciones lineales con un elevado número de incógnitas, tres por cada nudo de la estructura, que evidentemente sólo permite su tratamiento mediante ordenador. Ante la dilicultad de abordar el cálculo por métodos directos, en una primera época se emplearon métodos aproximados basados general mente en cálculo por analogías con placas equivalentes. Estos métodos permiten obtener una idea aproximada de los esfuerzos máximos en las bam ras horizontales de estructuras con formas basm tante regulares, pero ocasionan importantes erro res en la deteminación de los estuerzos sobre las diagonales proximas a los contornos o en zonas irregulares. Por otra parte la determinación de las deformaciones es bastante dudosa al no incluir debidamente el efecto del esfuerzo cortante.

Hoy dia no viene sentido aplicar los métodos de asimilación salvo en aproximaciones y predimen sionamientos. Para un dimensionado definitivo conviene recurrir al cálculo directo, que mediante programas adecuados puede ser ejecutado por la mayoría de los ordenadores de capacidad media.

Como se ha citado con anterioridad, una caracte ristica importante de las mallas espaciales es la capacidad para ser descompuestas en un gran número de elementos iguales o muy similares. Esto sugiere inmediatamente la prefabricación de los mismos que suelen ser simplemente barras y num dos. Mediante una adecuada estandarización estos elementos pueden ser fabricados de forma muy automatizada. Tanto los nudos como las barras son perfectamente aplables, tal como se encuentran a la salida de rabrica. Esto constituye una nueva ventaja porque, además de facilitar el almaw cenamiento, reduce sustanclalmente los costes de rransporte. Al citar la estandarización de los ele mentos conviene aclarar que en ningún modo implica la estandarización de las estructuras. Por el contrario, un sistema constructivo ingeniosamente disenado puede permitir con pocos elementos diferentes la construcción de las más varladas reallzaciones.

La automatización y sistematización de estas es. tructuras no se limita al proceso de fabricación de sus componentes sino que, en lo posible, se exviende al montale. Con tal fin los elementos suem len conectarse en obra mediante atomillacio por procedimientos muy repetitivos. El proceso que se utiliza habitualmente para el montale de mallas espaciales es consecuencia de la rigidez estrucural a que anteriormente se hacia referencia. Gene ralmente el ensamblaje de toda la cubierta se reaw llza a ple de obra. Posteriormente, mediante gruas, se eleva todo el conjunto o grandes rragm mentos del mismo hasta colocarlos en su empla zamiento delinitivo. Este proceso se caracteriza por su gran rapidez y proporciona una mayor se guridad de los operarios al reducirse al minimo las operaciones en altura.

Una consecuencia más de la estandarización es la gran adaptabilidad que presentan las mallas espaciales atomilladas para cambios o amplaciones futuras de las construcciones, e incluso para el traslado de toda una cubierta hasta otro lugar de emplazamiento. 
Finalmente como aspecto destacable de estas es. tructuras cabe citar su depurada estética. La armonía geométrica de un lado y el empleo del per. fil tubular por otro contribuyen a realizar una importante función ornamental que, frecuentemente, es buscada por el proyectista.

\section{Topologías básicas y elección de parámetros}

En la anterior descripción de las mallas espaciales se han considerado como más habituales las de doble capa, en las cuales los nudos se encuentran situados solamente en las dos superficies envolventes. Pueden generarse topologias que distribuyen sus nudos en diferente número de capas. Cabe citar las mallas de triple capa que han sido objeto de recientes investigaciones por parte del Prof. Makowski, con resultados interesantes acerca de su rigidez. Por ahora no han tenido gran difusión, ya que el gran numero de elementos y cone xiones que presentan hace poco rentable su empleo. También suelen proyectarse mallas de una sola capa. Estas si que presentan un notable interés económico, pero su empleo queda bastante restringido. Bajo el supuesto de que las uniones se comportan como nudos articulados las mallas monocapa sólo son estables si se distribuyen en supenficies de doble curvatura (cupulas) mediante reticulas triangulares. En cualquier caso no son económicamente aplicables a grandes luces por el riesgo que presentan de fenómenos de inestablim dad generalizada.

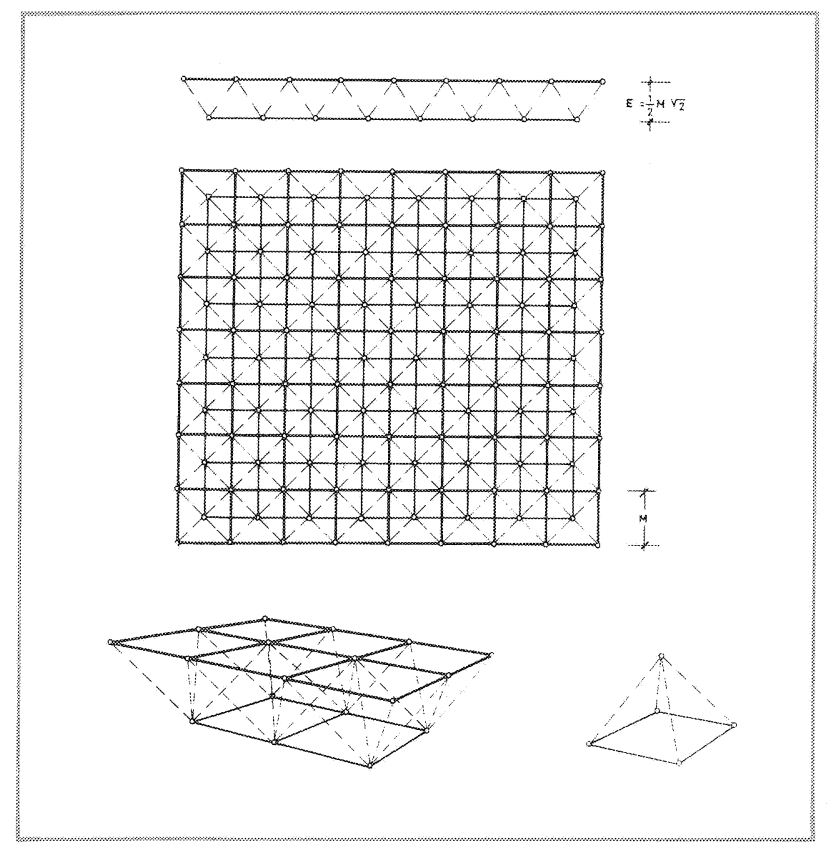

Fig. 1

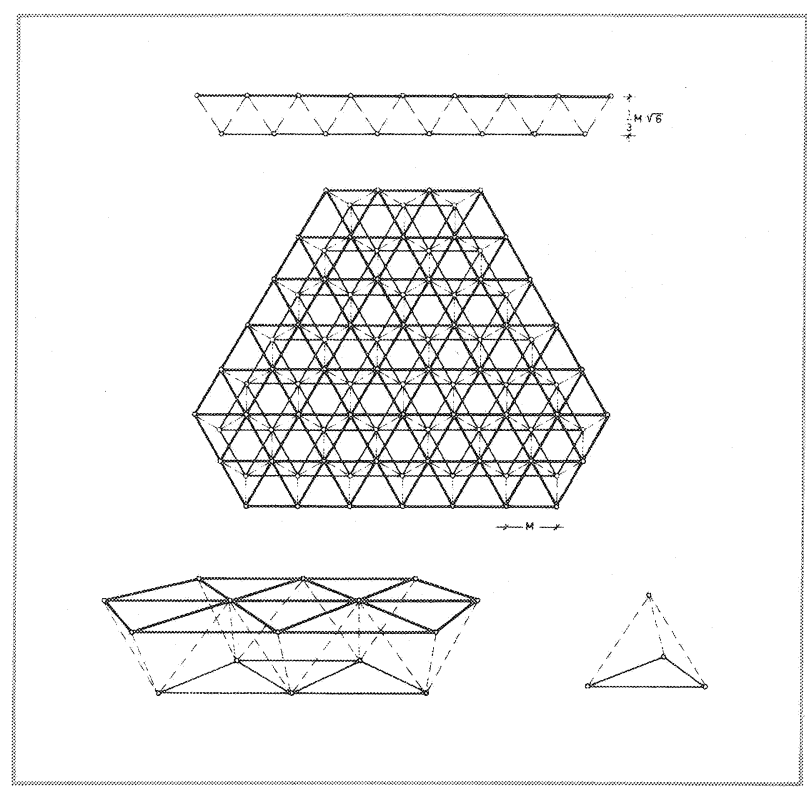

Fig. 2

En las aplicaciones usuales se emplean casi exclusivamente las mallas de doble capa. La limitada extensión de este documento nos impide una descripción detallada de todas las formas posibles de componer la geometria elemental de estas estructuras, no obstante se expondrán brevemente las topologias básicas más empleadas.

Como se habia citado anteriormente, la distribución de barras en el espacio se obtiene por yuxtaposición de poliedros estables. Los únicos polie dros regulares estáticamente determinados son el tetraedro y el octaedro; ambos dan lugar a la ge neración de las dos mallas más elementales.

La malla octaédrica, sin duda la más empleada, está generada por la repetición de semioctaedros - pirámides de base cuadrada, unos con el vértice hacia arriba y otros invertidos. En cada capa se forma una reticula cuadrada quedando los nudos de una de ellas sobre los centros de cuadrados de la opuesta. En cada nudo concurren ocho barras, cuatro horizontales y cuatro diagonales (figura 1).

La malla tetraedrica se obtiene por yuxtaposición de tetraedros. La retícula de cada capa es triangular existiendo tres direcciones preferentes de canalización de los esfuerzos (FIg. 2). Estas mallas son más rigidas que las octaédricas y producen una mejor distribución de estuerzos.

En general, para una misma aplicación, con la malla tetraédrica se consiguen esfuerzos notable mente inferiores a los que se producen con la octaédrica. También las flechas provocadas para 
Igualdad de capacidad resistente suelen ser menores. A pesar de esto las mallas tetraédricas son generalmente más caras porque, en el caso de aplicar la misma longitud elemental de barra, el número total de elementos requeridos para la construcción de la malla es notablemente superior. Como se expondrá más adelante, la densidad de nudos de una estructura es un factor decisivo en su economia. A titulo indicativo cabe citar que, por término medio, la malla tetraédrica puede re sultar del orden de un 20 a $30 \%$ más cara que la octaédrica, pero esto no debe considerarse en absoluto como una regla, ya que en determinadas aplicaciones puede incluso resultar más económica. En particular, en los casos de estructuras con planta hexagonal o triangular este tipo de malla puede resultar más adecuado por su mejor adaptación al contorno.

Partiendo de las dos topologías citadas pueden deducirse otras mediante cambios en la orientación de alguna de las reticulas, eliminación o adición de determinadas barras, etc. Algunas de estas derivaciones pueden incluso resultar más ecom nómicas en ciertas aplicaciones concretas, pero no se expondrán aquí por carecer de interés general.

Como en cualquier tipo de estructura, en las mallas espaciales concurren una serie de variables cuya incidencia sobre el coste puede ser importante. Algunas de ellas pueden quedar bastante impuestas por la funcionalidad del edificio a cubrir, como son la carga unitaria a soportar, la luz, etcétera. Sin embargo existen otras que, teniendo una repercusión trascendental sobre el coste, pueden ser elegidas con bastante libertad. Se ha citado ya la influencia que puede tener el tipo de malla. Mucha mayor trascendencia tienen los parámetros que la determinan, módulo y espesor.

Se entiende por módulo la longitud de cada una de las barras de la reticula, normalmente horizontales. El número de nudos y barras requerido por una estructura varia de forma inversamente proporcional al cuadrado del módulo. Análogamente a lo citado al comparar las dos topologias básicas, el incremento de la densidad de nudos repercute negativamente en el coste. Por ser el módulo la variable que mayor incidencia tiene en la densidad de nudos de la estructura, este parámetro resulta ser el de mayor repercusión económica. Valores inferiores a los $2 \mathrm{~m}$ son casi siempre antiecom nómicos y sólo cabe aplicarlos en condiciones especiales o en estructuras de reducidas dimen. siones con carácter preferentemente ornamental. Cuando las dimensiones generales y las luces son importantes conviene acercarse a los $3 \mathrm{~m}$ de modulación. Por encima de estos valores la estructu- ra puede resultar todavia más económica, pero normalmente no interesa demasiado su utilización desde una perspectica global porque los sistemas habituales de cerramiento, a base de chapas, sandwich, etc., no permiten mayores separaciones entre apoyos. Módulos muy superiores obligarian a una estructura auxiliar para el apoyo del elemento de cierre y el coste total podria salir perjudicado.

En cuanto al espesor de la malla, también tiene una incidencia apreciable en el coste, ya que los esfuerzos originados en una determinada malla son inversamente proporcionales al valor de este parámetro. Con anterioridad se ha citado el orden de magnitud que adquiere el cociente espesor/luz para las utilizaciones comunes en cubiertas planas. En cuanto a su relación con el módulo, el espesor tambien debe estar comprendido entre ciertos límites. El cociente espesor/módulo norm malmente suele estar comprendido entre 0,5 y 1 . Relaciones inferiores provocan esfuerzos exces:vos en las barras diagonales, mientras que las superiores tienden a aumentar la esbeltez de estas barras obligando a dimensionamientos excesivos por pandeo. En general, en estructuras de grandes luces con esfuerzos importantes conviene que el espesor se aproxime al valor del módulo, siempre que sea compatible con las posibles limitaciones que por otras razones puedan imponerse al volumen de la cubierta.

En la figura 3 puede apreciarse la influencia combinada del módulo y espesor sobre el coste. Estas tendencias serian aplicables a estructuras concretas, en el supuesto de que se mantengan constan-

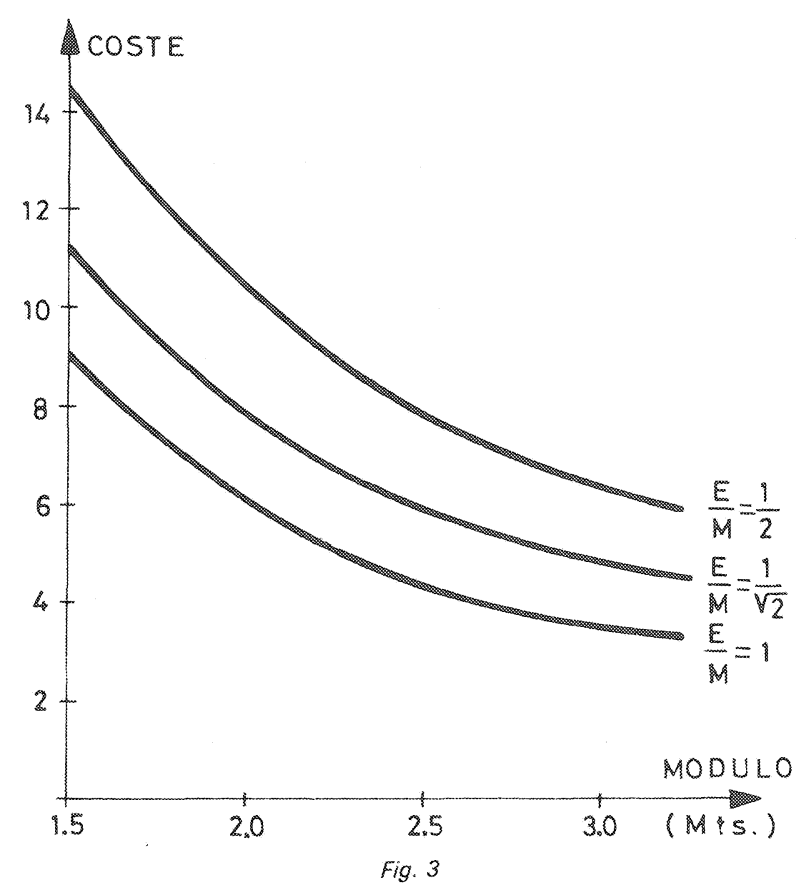


tes las demás variables. También conviene aclarar que en lo que respecta al espesor este grálico solo es vallido para los casos de grandes luces en cubiertas planas. En los casos de estructuras con reducidos esfuerzos la incidencia del espesor en el coste en mucho menos apreciable.

\section{Sistemas constructivos}

En las mallas espaciales ordinarias, las barras se conectan entre si por medio de una pieza especial que constituye el núcleo del nudo. Las conexio nes presentan unas caracteristicas pecullares muy diferenciadas de las que se producen en las estructuras planas. El nudo debe permitir la conm currencia de un elevado número de barras, ocho o nueve frecuentemente, algunas de ellas bajo direcciones muy diversas en el espacio; pero además debe proporcionar el cumplimiento de unas condi clones técnicas aceptables dentro de un diseño económicamente viable.

Las propledades deseables más interesantes que, a nuestro julcio, deben reunir los sistemas de co nexión son:

a. Capacidad para adaptarse a cualquier distribución geometrica de barras en el espacio.

b. Sencillez de montaje e Independencia en el or den de ensamblaje de los elementos, cualquie ra que sea la topologia.

c. Fiablidad de la estructura.

d. Optimo aprovechamiento de los materiales.

e. Comportamiento acorde con la hipótesis de cálculo.

1. Bajo coste de mantenimiento.

Existen muchos sistemas para la construcción de este tipo de estructuras, con disenos muy variam dos, pero no todos se aproximan suficientemente al cumplimiento de las propiedades indicadas. En una primera época se emplearon sistemas solda dos en obra, pero han caido en desuso por tener dos graves inconvenientes. Por un lado la calldad de las soldaduras es dudosa y sólo mediante costosas inspecciones radiográflcas puede conseguir se una garantía suficiente. Por otro lado el proce so de montale resulta excesivamente prolongado y costoso, no quedando compensado por el inferior coste de los componentes que salen de fábrica.

La propiedad citada de un comportamiento acorde con las hipótesis de cálculo, para los programas normales de análisis de mallas, implica que la unión debe asemejarse a una rotula pura. Esto supone una rigidez cónica baja combinada con una rigidez axil elevada. En general los sistemas atomillados se aproximan bastante a la reducida rigidez conica, pero no todos aportan una rigidez axil suficiente. La conexión atornillada presenta siempre una cierta discontinuidad con mayor o menor flexibilidad localizada y generalmente con un comportamiento no lineal. Si la rigidez axil es muy bala, la distribución de esfuerzos obtenidos por el cálculo puede discrepar notablemente de la que realmente se producirá en la estructura, pero además las deflexiones reales originadas bajo las cargas de trabajo pueden ser muy superiores a las determinadas en el cálculo. Cualquier sistema de conexión debe ser ciudadosamente ensayado tanto a nivel de nudos como de pequenas estructuras con el lin de conocer su comportamiento real. Pueden considerarse satistactorios los sistemas que por termino medio producen deflexiones gla bales no superiores en un $10 \%$ a las determinadas por cálculo balo la hipótesis de articulaciones puras.

No es objeto de este trabalo la descripción de los distintos sistemas que se encuentran en el merca. do, no obstante cabe citar aqui el sistema MERO diseñado por el Dr. Mengeringhausen, que fue uno de los precursores del empleo de las mallas espaciales. A pesar de que sus primeras elecuciom nes datan de hace cuarenta años, este sistema presenta codavia una completa actualidad siendo, sin duda, el más difundido hasta el momento. Bám sicamente está constituido por nudos esfericos y barras tubulares que se atornillan axllmente sobre los nudos por medio de un ingenioso dispositivo de conexión. Con esta disposición consigue un razonable equilibrio entre las propledades citadas anteriormente.

Todo sistema de conexión debe ser capaz de som portar los diferentes ordenes de esfuerzos que pueden darse dentro de la gama de estructuras en que desee aplicarse. Resulta conveniente estanda rizar los componentes que lo integran dentro de diversos niveles de solicitación con un escalona. miento tal que no provoque un excesivo desaprom vechamiento de los materiales. La capacidad má. xima de los elementos estandarizados suele oscim lar de unos a otros sistemas entre 50 y 100 t. En la mayoria de ellos las barras normalmente se fabrican con perfil tubular y con acero de calidades comprendidas entre A-37 y A-52. Sus dámetros suelen oscilar entre unos 40 y $150 \mathrm{~mm}$ y los espe sores entre unos 2 y $8 \mathrm{~mm}$. Evidentemente pue den conseguirse elementos más resistentes con dimensionamientos superiores para las aplicaciones que se salgan de la gama estandarizada. 


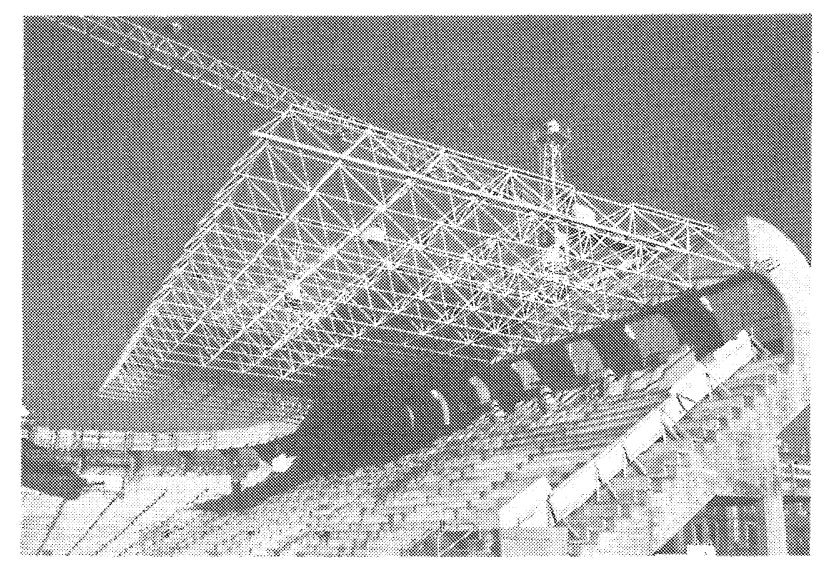

Los nudos suelen presentar muy variados diseños según los diferentes sistemas de conexión, pero en cualquier caso deben estar capacitados para recibir las tracciones y compresiones de las barras concurrentes. Cada diseno exigirá unas dimensio nes minimas dependientes del nivel de esfuerzos para el que está capacitado. Es conveniente que el tamaño del nudo sea reducido con el fin de evitar posibles tenómenos de inestabilidad por ge neración de rótulas plásticas. Las formas compacm tas son en este sentido las más convenientes.

También en el tamaño de los tornillos deben buscarse los minimos compatibles con la resistencia precisa, de modo que se eviten posibles interfe rencias de barras en las proximidades del nudo. Esto lleva frecuentemente al empleo de tornillos de alta resistencia. En general, en el extremo de cada barra se sitúa una pieza que transmite el esfuerzo del tubo al tornillo. La conexion de dicha pleza al tubo se realiza por soldadura en taller $y$ su proceso debe ser cuidadosamente controlado bien sea mediante ensayos no destructivos o por métodos destructivos aplicados por muestreo.

Las dimensiones de los elementos, tal como salen de fábrica, suelen ser relativamente reducidas, no muy superiores a $3 \mathrm{~m}$ de longitud. Esto facilita la

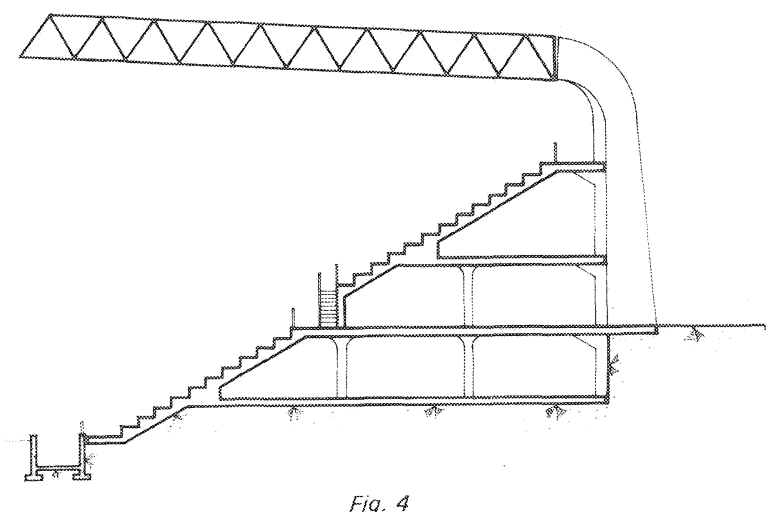

aplicación de protecciones anticorrosivas mucho más eficaces que las empleadas sobre las estructuras convencionales por aplicación de pinturas en obra. Entre las direrentes formas de suministro habituales de las mallas espaciales cabe citar como más eficaces la galvanización en caliente y el pintado mediante resinas polimerizadas en homo.

Si bien pueden existir diferencias muy notables de coste entre unos y otros sistemas dependiendo del diseño concreto de cada uno, en cualquier caso el nudo, entendiendo por tal el conjunto de elementos que materializan la unión, es normalmente la parte más cara de estas estructuras. Las plezas que lo componen serán más o menos complicadas, pero siempre aportan un valor añadido muy superior al de los tubos. De aquil se deduce inmediatamente el interes de reducir en lo posible el numero de nudos requeridos para formar una estructura.

Con el fin de cifrar lo expuesto en el párrafo ante rior cabría hacer un desglose de los componentes del coste en estas estructuras. Esta descomposición puede oscilar mucho de unos a otros sistemas e, incluso, de unas a otras aplicaciones con con el mismo sistema; no obstante, con carácter orientativo, nos permitiremos presentar unos valores medios que aproximadamente serian:

Tubo: 25 - 35\% (supone el 90\% del peso total). Nudos (componentes y fabricación): $35-45 \%$.

Protección y pintura: $10-15 \%$.

Montaje: $15-20 \%$.

\section{Tipologías para cubiertas de estadios}

Las cubiertas para tribunas de estadios deportivos están sujetas a ciertos condicionamientos particulares que merecen ser destacados porque tienen una repercusión directa sobre las formas estructum rales capacitadas para soportarlas. En primer lugar, el contorno próximo al terreno de juego debe estar libre de columnas que pudieran impedir la correcta visión del espectáculo por parte del púm blico. Esto obliga a la aplicación de estructuras con grandes voladizos, frecuentemente entre 20 y 30 metros.

En segundo lugar estas estructuras suelen ser de tipo ligero. Las cargas exteriores se limitan a la acción de nieve y viento, ya que se trata de cubientas no transitables, y el peso del cerramiento suele ser minimo, correspondiendo al de una simple chapa o lámina traslúcida. Por otra parte, al 


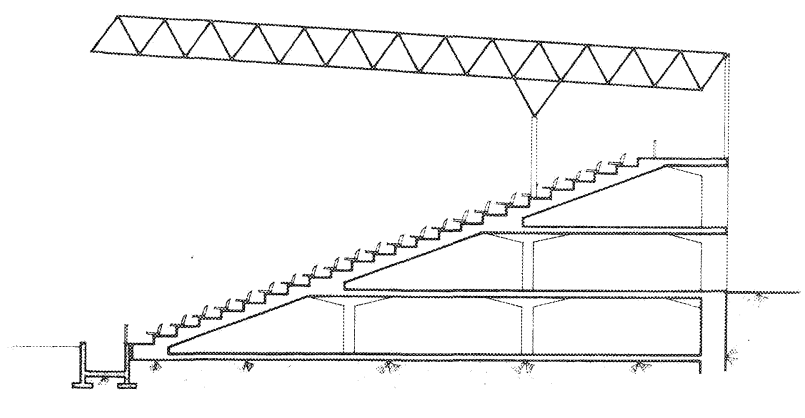

Fig. 5

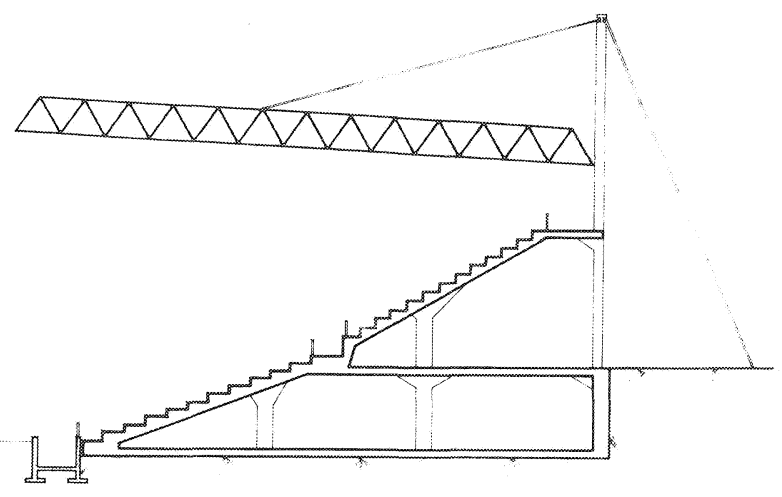

Fig. 6

tratarse de construcciones abiertas pueden quedar sometidas a fuertes presiones de viento en sentido tendente a levantar la cubierta. Dado que la carga permanente es reducida, en muchos casos las hipótesis de carga invertida superan en solicitaciones a las de carga directa. Casi todos los elementos sufren inversiones de esfuerzos. Las estructuras metálicas convencionales a base de vigas, bien sean de alma llena o de celosía, en situación de carga directa, tienen comprimido su cordón inferior. En inversión de esfuerzos el cordón comprimido sería el superior. Para evitar posibles fenómenos de inestabilidad lateral se requiere en general una disposición de barras de arriostramiento en ambos cordones, sobre todo en el inferior, que complica estas estructuras y las encare

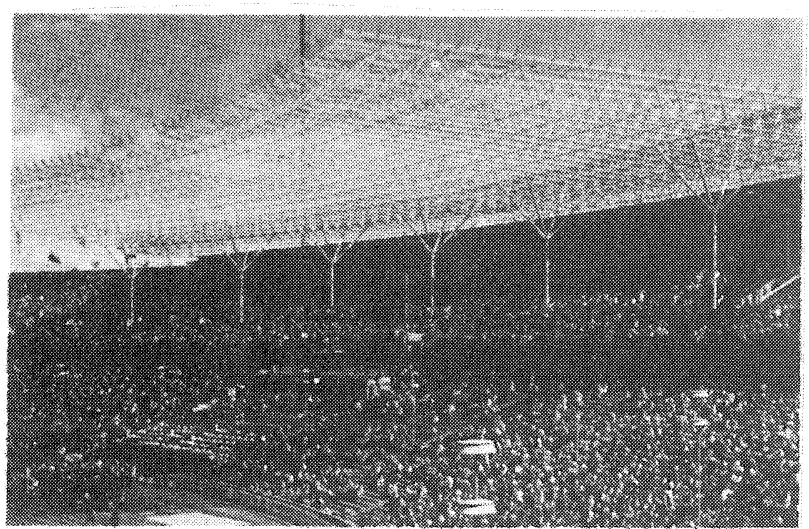

ce. Por el contrario, en las mallas espaciales ambas capas quedan perfectamente arriostradas por la propia constitución de la estructura sin requerir elementos adicionales.

Otro aspecto que favorece el empleo de estructuras espaciales en estas aplicaciones es la simplicidad y rapidez de montaje. Las estructuras convencionales requieren un determinado orden de montaje de sus elementos, empezando por los más resistentes, celosias o vigas largas a veces poco rigidas, que deberian colocarse completos, a veces teniendo incluso que recurrir a apeos provisio nales. Todo ello se complica por las peculiares caracteristicas de los graderios y el importante desnivel entre la cubierta y los mismos.

Dependiendo de las posibilidades de acceso de gruas y de sus capacidades, las mallas pueden ser fragmentadas por donde convenga. Cada fragmento se ensambla a pie de obra y tiene por si solo la rigidez suficiente para ser izado y coloca do en su emplazamiento. Por otra parte las barras perpendiculares a las de la dirección principal, que en el trabajo ordinario de la estructura quedarian sin solicitación (normalmente las paralelas al campo), pueden durante el montaje realizar un trabajo preponderante permitiendo muy diversas posibilidades en cuanto al orden de composición de la estructura total. Todo ello da una gran llexibilidad al proceso de montaje, y en los casos de mallas atornilladas permite el desarrollo del mismo con gran rapidez. Como ejemplo cabe citar la estructura de la nueva cubierta del Estadio de Balaidos, en Vigo. Su superficie en planta es de unos 6.000 metros cuadrados y fue montada por un equipo de sólo cinco hombres en treinta y dos dias laborables.

La solución más simple de aplicación de mallas espaciales a estas cubiertas consiste, como es 10 gico, en hacerlas trabajar preferentemente en la dirección perpendicular al campo, a modo de sim ple viga en voladizo. Las disposiciones concretas podrán ser diversas. En la figura 4 se muestra una de ellas en la cual la malla se empotra directamente en la sustentación. Esta disposición ha sido empleada en las tribunas recientemente remodeladas del campo de fútbol de San Mamés, en Bilbao.

Con esta disposición puede llegarse hasta vuelos del orden de los 25 a $30 \mathrm{~m}$. Por encima de estos valores los esfuerzos se incrementan excesivamente encareciendo la estructura fuera de los márgenes razonables. No obstante cabe citar, como datos orientativos, que estructuras para cubiertas con voladizos del orden de los $25 \mathrm{~m}$ realizadas 
con malla espacial presentan un peso proplo de 15 a $20 \mathrm{~kg} / \mathrm{m}^{2}$, lo que demuestra la extraordinaria ligereza de estos sistemas.

Conviene tener presente que, a igualdad de nive les de esfuerzo, la luz equivalente de una estrucura biapoyada será el doble de la del voladizo. La relacion entre espesor y luz, que para estructuras apoyadas en todo el contorno se cilraba en el orden de $1 / 20$, en estas disposiciones no debe ser inferior a $1 / 1361 / 14$ con el fin de no generar excesiva flecha en el extremo. En cualquier caso estas relaciones son bastante lavorables respecto de las que cabe obtener con otros sistemas estructurales. Por otro lado las mallas espaciales se prestan muy bien a la creación de contraflechas, con objeto de que la malla quede perfectamente plana bajo la acción de las cargas permanentes. Para ello basta con diferenclar muy ligeramente las dimensiones de las barras, acortando en origen las traccionadas y alargando las comprimidas. En la aplicación a voladizos se acortarian las superiores de la dirección perpendicular al contomo empotrado, y se alargarian las inferiores.

Cuando se requiere cubrir mayores secciones de graderio que las que permite el voladizo simple puede recurrirse a otras disposiciones que faclliten algún punto de sustentación más próximo al

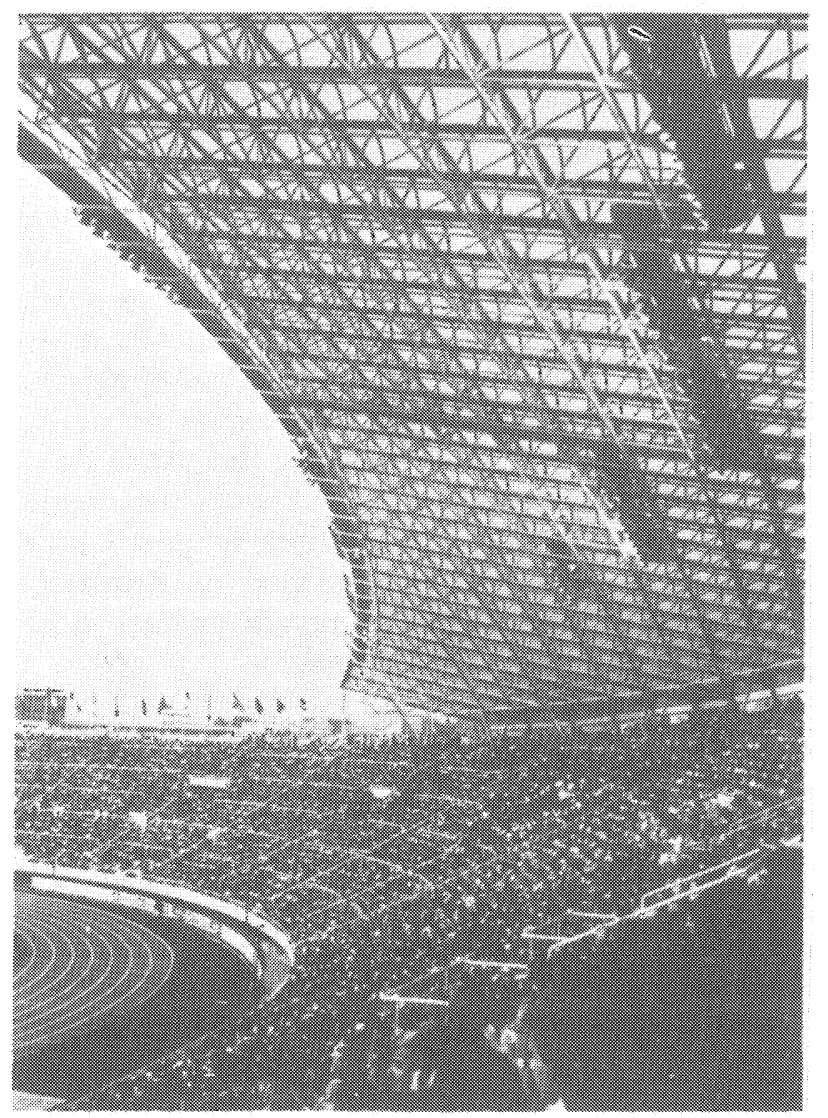

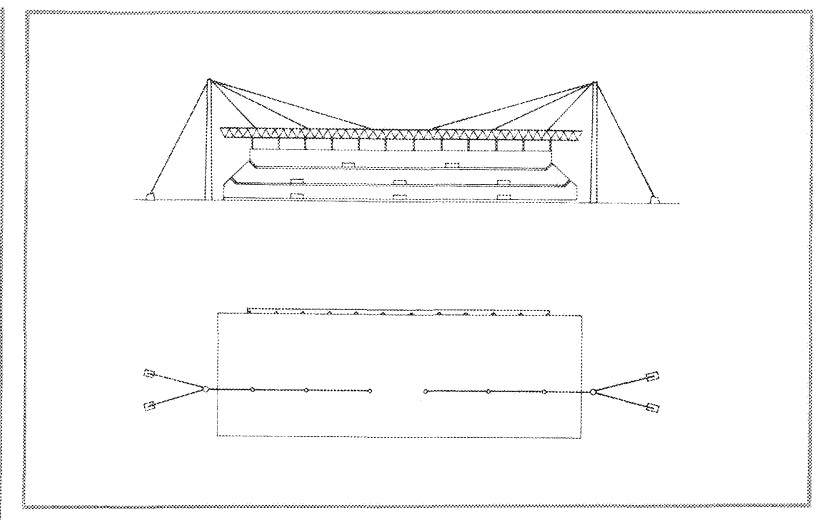

Fig. 7

borde libre de la estructura. En la figura 5 se muestra una solución a base de dos apoyos en cada pórtico, quedando los delanteros comprimidos y los traseros traccionados. La figura 6 muestra una disposición que proporciona a la malla un punto de apoyo delantero mediante un tirante exterior. La primera de estas soluciones supone la existencia de columnas dentro del campo visual de algunos espectadores, pero este inconveniente puede quedar compensado por su mayor economia.

Como se recordară, una de las propiedades inte resantes que presentan las mallas espaciales es la de permitir una notable reducción del número de apoyos, propiciando además una distribución de los mismos mucho más flexible. Aplicando debidamente esta propledad a la disposición de apoyo delantero, mediante columnas, se puede minimizar el inconveniente citado llegando a soluciones muy satisfactorias. Un caso muy representativo de esta disposición es el de las cubiertas del Estadio de Berlin. Cada cubierta tiene una longitud aproximada de $150 \mathrm{~m}$ y anchura de $57 \mathrm{~m}$, y se sustenta únicamente en 6 apoyos delanteros equidistantes unos $25 \mathrm{~m}$ entre si. La malla espacial constituyente de esta cubierta tiene una topología muy bien seleccionada. Con el in de adaptarse a una plan ta en forma de sector de anillo circular y tratando de reducir en lo posible el número de nudos diferentes, presenta una malla octaédrica distorsionada de forma que las barras horizontales se dispo nen radial y circunferencialmente. Para poder mantener constantes los ángulos de incidencia so bre los nudos, las pirámides de generación de la topología son todas semejantes y tienen un tamam ho proporcional al radio en que se encuentran situadas. En consecuencia, no sólo las barras circunferenciales, sino también las radiales y el es pesor se van reduclendo a medida que se aproximan al centro de curvatura. Como consecuencia, las capas exteriores de la malla no son paralelas. 

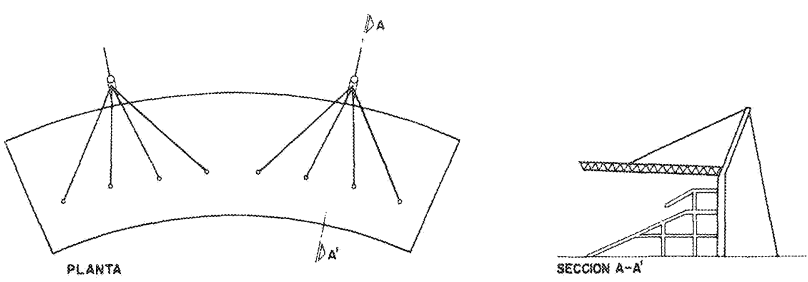

tig 8

En las disposiciones constructivas precedentes el trabajo estructural se orienta fundamentalmente en una sola dirección, lo que en cierto modo implica el mal aprovechamiento de una de las ventajas que ofrecen las estructuras espaciales. Cabria preguntarse si en tal caso tiene sentido la utilizacion de las mallas para estas aplicaciones. Los aspectos citados anteriormente, ligereza, estructura rigida muy arriostrada, flexibilidad de montaje, \|fbertad en la disposición de los apoyos, etc., son suficientes para justificarlo incluso desde una perspectiva puramente económica. El ejemplo del Estadio de Berlin aporta alguna mejora en el sentido de que por lo menos, en la zona próxima a los apoyos, se ha aprovechado el trabajo de las barras circunferenciales precisamente para conseguir la separación de los mismos.

Cuando se pretenden obtener grandes voladizos, evitando totalmente las columnas intermedias, es preciso buscar otro tipo de soluciones que permi

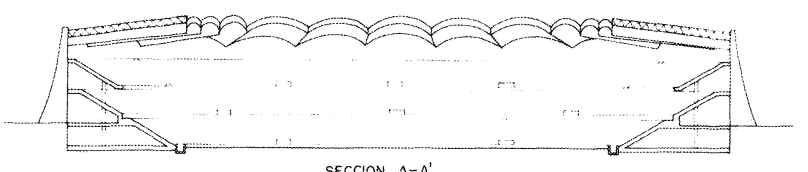

$\triangle E C C I O N A-A^{\prime}$

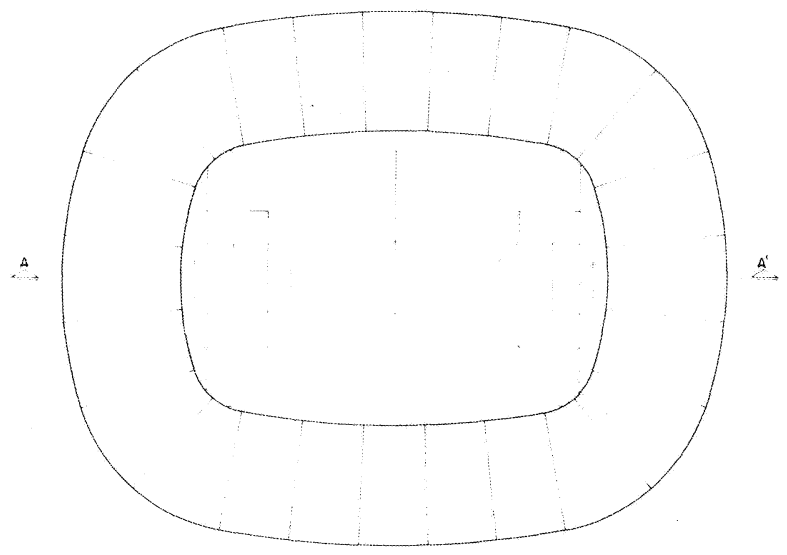

Fig. 9 tan una mejor utilización de las mallas. En partim cular, una buena orientación en este sentido constituyen aquellas tipologias encaminadas a combinar el trabajo en sentido longitudinal (paralelo al borde del terreno) con el transversal que es el que ordinariamente trabaja en voladizos puros. Una solución precursora de este trabajo, si bien no fue ejecutada mediante malla espacial por su anterio ridad al empleo de estas estructuras, es la que se adoptó para la sustentación de la cubierta principal del Estadio de San Mamés. Un gran arco de 118 metros de luz dispuesto paralelamente al campo y apoyado fuera de los bordes laterales de la tribuna sustenta la cubierta que cuelga de aquél mediante pendolones.

Cualquier tipología aplicable a la construcción de grandes cublertas, mediante sistemas estructurales más convencionales, puede ser desarrollada mediante mallas espaciales y probablemente con grandes ventajas por las pecullares propiedades de estas estructuras. Formas estructurales tales como bóvedas y cúpulas son perfectamente desa rrollables con mallas espaciales. La consecución de una curvatura simple se puede lograr por distorsión de una malla octaédrica sin más que modificar las barras de una de las direcciones de la malla. Asi, para la obtención de una bóveda basta alargar las barras de una de las direcciones de la capa superior o acortar las de la misma dirección en la inferior. La aplicación de una distorsión similar en las dos direcciones principales daría lugar a la generación de un casquete esférim $\mathrm{co}$, si bien existen otras topologías diversas para la consecución de cúpulas. Con estas disposiciones las mallas permiten abordar razonablemente luces muy superiores a las de las cubiertas plam nas. El comportamiento conjunto ya no es el de una simple placa plana que flexiona en dos direcciones, sino que se asimila más al de una cáscara con sus correspondientes componentes de solicitación axil dentro de la superficie generatriz de la misma. Con estas disposiciones constructivas el cociente entre espesor y luz libre adquiere unos valores mucho más reducidos que los expuestos para las cubiertas planas, pudiendo llegarse a valores de $1 / 50$ e incluso inferiores.

Un caso muy singular y probablemente el más significativo de la capacidad de empleo de las mallas espaciales es el de las cubiertas sobre las tribunas del Estadio de Split, en Yugoslavia. Estas cubiertas han sido diseñadas bajo la perspectiva de hacer trabajar a la malla en dirección paralela al terreno de juego. Se trata de sendos fragmentos de bóvedas con generatriz inclinada y seccionados por unos planos inclinados que dan lugar a contornos elipticos. Cada cubierta en planta pre- 
Informes de la Construcción

senta una forma similar a un gajo de naranja. En su punto de máxima anchura el vuelo es de $45 \mathrm{~m}$, lo que hublese sido excesivo para un trabajo en voladizo simple. La solucion adoptada ha consistido en apoyar la maya en todo su contorno exterior haciendola trabajar en forma de bóveda con una separación creciente que en sus extremos llega a $215 \mathrm{~m}$. Siendo el espesor de la malla de 2,30 , la Iracción espesor/luz resulta ser 1/90. Esta estructura ha sido construida mediante el sistema MERO y contiene del orden de 3.500 nudos y unas 13.000 barras distribuidas en una malla octaédrica de $3 \mathrm{~m}$ de módulo. El peso propio de la estructura es de $40 \mathrm{~kg} / \mathrm{m}^{2}$. La ejecución de una cáscara de estas dimensiones mediante sistemas constructivos tradicionales hubiera sido muchisimo más pesada, requiriendo un montaje más lento $y$ costoso.

En la misma linea de pretender encauzar los esm fuerzos según la dirección longitudinal de las cubiertas pueden proyectarse diversas tipologias, al gunas de las cuales se citan a continuación a titum lo de ejemplo.

Cada vez se emplean en mayor proporción las estructuras suspendidas por cables para la consecución de grandes vanos $\sin$ apoyos.

Bajo esta idea cabria sustentar toda la parte de lantera de las cubiertas de estadios mediante tirantes. En las figuras 7 y 8 se presentan dos sa luciones de este tipo. La primera de ellas sería aplicable a los casos en los que tanto los mástim les como los anclajes opuestos de los tirantes puedan situarse por los costados de la cubierta, mientras que la segunda se emplearia con estos elementos situados detrás del graderio. No obstante, en las soluciones a base de estructura atim rantada hay que tener presente el problema suscitado por una posible inversión de esfuerzos que tenderia a levantar la cubierta. En los casos en que esto sea posible cabe recurrir al diseño de alerones o deflectores del viento que lo impidan.

Otra gama de posibilidades se plantea ante la ream lización de cubiertas tales que deban cerrar todo el contorno exterior del estadio. En el supuesto de que dicho contorno tenga una forma sensible mente circular, estos casos se prestarian a la realización de grandes cúpulas incompletas a falta de un circulo central que dejaría sin cubrir el terreno de juego. En tal caso las barras circunferenciales trabajarian como anillos comprimidos colaborando con las radiales. Tal vez esta situación pueda considerarse un tanto ficticia, porque normalmente los contornos exteriores de los estadios no son circulares y tampoco interesa que el espacio no cublerto tenga forma circular. Sin embargo derivándose de esta disposición caben soluciones adaptables a las formas concretas de los estadios deportivos. En la figura 9 se representa una posible derivación de la idea anterior. La cubierta se ha dividido en una serie de sectores cada uno de los cuales es una especie de bóveda distorsiona. da, cuya superficie constituiria un sector cónico. Las generatrices de las bóvedas tienen una inclinación ascendente para que, cuando por acción de las cargas el borde de la cubierta tienda a descender, entren en acción los elementos estructurales parelelos al contorno interior, comportándose como anillos comprimidos. De este modo se garantizarian unos empujes horizontales entre las bóvedas contiguas de modo que pueden trabajar en la dirección de sus arcos.

Indudablemente las cubiertas para estadios se prestan a la aplicación de muy diversas tipologias que pueden ir desde la formas estructurales más simples hasta las de más sofisticada fantasia. Las interesantes propiedades que presentan las mallas espaciales, reiteradas a lo largo del presente articulo, hacen de estas estructuras una herramienta extraordinariamente capaz para materializar las diferentes soluciones estructurales proyectadas. Una utilización racional de las mismas, en combinación con los restantes elementos estructurales del proyecto, puede reportar importantes beneficios tanto económicos como funcionales. Aspectos tales como la selección del tipo de malla a emplear, determinación de los parámetros más convenientes, localización de los puntos de apoyo, etc. de ben ser objeto de un detallado estudio y fruto de colaboración entre el director del proyecto y el especialista en el tratamiento de las mallas espaciales. 